\title{
Contribución al estudio de la hepatitis infecciosa y de la cirrosis hepática*
}

\author{
por \\ Mario Miranda* ${ }^{*}$ \\ (Recibido para su publicación el 6 de noviembre de 1956)
}

En los últimos años el número de pacientes con hepatitis infecciosa y cirrosis hepática que hemos observado en el Hospital San Juan de Diós es tal que podemos decir que estas dos afecciones son las más frecuentes entre las enfermedades hepatobiliares.

También en el Departamento de Anatomía Patológica se ha confirmado un marcado predominio del tipo de cirrosis post-necrótica con respecto a la cirrosis portal.

El presente trabajo tiene como finalidad:

a) Estudiar las características clínicas y de laboratorio de los casos de hepatitis infecciosa y cirrosis hepática.

b) Dar a conocer la frecuencia de los diferentes tipos de cirrosis hepática en un grupo de casos fallecidos a causa de esta afección.

:) Hacer algunas consideraciones epidemiológicas sobre estas dos entidades en Costa Rica.

\section{MATERIAL Y METODOS}

El presente estudio, está basado en una revisión de los pacientes con hepatitis infecciosa y cirrosis hepática ingresados al Hospital San Juan de Dios durante los años 1950, 51, 52, 53 y el año 54 hasta el mes de septiembre inclusive. Se han tomado en cuenta también pacientes de la sección de Pediatría. Se han excluído los casos en que el diagnóstico de hepatitis infecciosa o cirrosis hepá-

* El material clínico de este trabajo fue presentado como parte del tema oficial al XXIV Congreso Médico Nacional de Costa Rica.

$\therefore$ Asistente del Servicio de Medicina $\mathrm{N}^{\circ} 2$ del Hospital San Juan de Dios. 
tica no estaba adecuadamente fundamentado por la clínica o el laboratorio. Se estudian 145 casos de hepatitis infecciosa y 95 casos de cirrosis hepática. métodos:

Las determinaciones de laboratorio se hicieron mediante los siguientes

1. Pruebas de floculación

a) Oro coloidal: se realizó según la técnica de Mac-Lagan (cit. en Ducci (2) ) con sol citratado.

b) Turbidez del timol: hecha mediante la técnica de MacLagan de comparación visual (cit. en Ducci (2) ).

c) Rojo coloidal: según Ducci (2).

d) Hanger: según DucCI (2)

e) Agua bidestilada: según Ducci (2).

2. Bilirrubinemia:

Se realizó según la técnica de Malloy y Evelyn (4).

3. Colesterolemia:

Según la técnica de Bloor (1).

4. Fosfatasas alcalinas:

Se realizó según el método de Shinowara, Jones y Reinhart (8).

5. Proteinemia:

Se realizó mediante la técnica de Greenberg (cit. en pág. 570 del MANUAL Lumetron (7) ).

\section{RESULTADOS}

Desde el año 1950 hasta 1954 ingresaron al Hospital San Juan de Dios un total de 127.423 pacientes provenientes de todo el país; de este total de enfermos, en 145 casos ( 71 hombres y 74 mujeres) se diagnosticó una hepatitis infecciosa y en 95 ( 55 hombres y 40 mujeres) una cirrosis hepática. La distribución por años de estos casos está representada en el cuadro 1.

\section{CUADRO 1}

Distribución por años de los casos de hepatitis infecciosa y cirrosis hepática

\begin{tabular}{r|c|c|c|c|c}
\hline \multirow{2}{*}{ Año } & $\begin{array}{c}N^{\circ} \text { de } \\
\text { pacientes }\end{array}$ & \multicolumn{2}{|c|}{ Hepatitis infecciosa } & \multicolumn{2}{|c}{ Cirrosis hepática } \\
\cline { 3 - 6 } & No casos & $\%$ & $N^{\circ}$ casos & $\%$ \\
\hline \multirow{2}{*}{ TotAL } & 127.423 & 145 & 0,113 & 95 & 0.074 \\
1950 & 20.616 & 9 & 0.033 & 9 & 0,033 \\
1951 & 27.525 & 31 & 0.11 & 13 & 0,065 \\
1952 & 28.764 & 45 & 0.16 & 21 & 0.069 \\
1953 & 30.793 & 24 & 0,07 & 23 & 0.074 \\
1954 & 19.725 & 36 & 0.18 & 24 & 0,12 \\
\hline
\end{tabular}


Como puede verse en el cuadro 1 , el mayor porcentaje tanto de hepatitis como de cirrosis, se obtuvo en el año de 1954, a pesar de que el número de pacientes corresponde a los ingresos de los primeros ocho meses.

En cuanto a la distribución geográfica, hemos observado que el mayor número de casos corresponde a la provincia de San José, muy probablemente por ser la más densamente poblada: el mayor número de casos en esta provincia se observó en los cantones de Mora, Santa Ana y Puriscal, siendo los situados al norte de la Meseta Central los que muestran la más baja incidencia. La distribución por cantones de los casos de hepatitis infecciosa y cirrosis hepática en la provincia de San José se puede ver en el cuadro 2.

\section{CUADRO 2}

Distribución por cantones, en la provincia de San José, de los casos de hepatitis infecciosa y cirrosis hepática presentados durante el periodo 1950-1954

\begin{tabular}{|c|c|c|c|c|c|}
\hline \multirow{2}{*}{ Cantón } & \multirow{2}{*}{$\begin{array}{c}\mathrm{N}^{\bullet} \\
\text { habitantes }\end{array}$} & \multicolumn{2}{|c|}{ Hepatitis infecciosa } & \multicolumn{2}{|c|}{ Cirrosis hepática } \\
\hline & & $\mathrm{N}^{\circ}$ casos & $\%$ & $\mathrm{~N}^{\circ}$ casos & $\%$ \\
\hline TOTAL & 223.461 & 88 & 0,039 & 35 & 0,016 \\
\hline Central & 121.769 & 39 & 0,032 & 14 & 0,011 \\
\hline Mora & 8.698 & 15 & 0,174 & 7 & 0,080 \\
\hline Puriscal & 19.942 & 9 & 0,045 & 4 & 0,021 \\
\hline Escazú & 8.029 & 6 & 0,075 & 1 & 0,012 \\
\hline Santa Ana & 6.593 & 4 & 0,061 & 6 & 0,091 \\
\hline Curridabat & 4.882 & 1 & 0,020 & 0 & 0,000 \\
\hline Desamparados & 17.684 & 6 & 0,034 & 1 & 0,005 \\
\hline Goicoechea & 22.642 & 5 & 0,022 & 1 & 0,004 \\
\hline Moravia & 6.421 & 2 & 0,031 & 1 & 0,015 \\
\hline Coronado & 6.801 & 1 & 0,015 & 0 & 0,000 \\
\hline
\end{tabular}

En cuanto a la edad, la hepatitis infecciosa presentó la incidencia más alta en el decenio de 20 a 29 años, mientras que la cirrosis hepática fue más frecuente en personas de 50 a 59 años como puede verse en el cuadro 3.

\section{HEPATITIS INFECCIOSA}

\section{CuAdRo CLÍNico}

En vista de que muchas de las anamnesis que se recogen en la admisión de enfermos son incompletas, el análisis de los síntomas clínicos y de los signos físicos que se apuntan en el cuadro 4, recoge sólo los datos más importantes de la enfermedad. 


\section{CUADRO 3}

Distribución por grupos de edades de los casos de bepatitis infecciosa $y$ cirrosis bepática

\begin{tabular}{|c|c|c|}
\hline \multirow{2}{*}{ Grupos de edades } & \multicolumn{2}{|c|}{ Número de casos } \\
\hline & Hepatitis infecciosa & Cirrosis hepática \\
\hline 0 a 9 & 31 & 7 \\
\hline 10 a 19 & 20 & 6 \\
\hline 20 a 29 & 32 & 2 \\
\hline 30 a 39 & 29 & 10 \\
\hline 40 a 49 & 17 & 16 \\
\hline 50 a 59 & 13 & 24 \\
\hline 60 a 69 & 3 & 19 \\
\hline 70 a 79 & 一 & 9 \\
\hline 80 o más & $一$ & 2 \\
\hline
\end{tabular}

\section{CUADRO 4}

Frecuencia de los sintomas clínicos y los signos físicos en 145 casos de bepatitis infecciosa

\begin{tabular}{|c|c|c|c|c|c|}
\hline \multicolumn{3}{|c|}{ Síntomas clínicos } & \multicolumn{3}{|c|}{ Signo's físicos } \\
\hline Síntoma & $\mathrm{N}^{\circ}$ casos & $\%$ & Signo & $\mathrm{N}^{\circ}$ casos & $\%$ \\
\hline Ictericia & 130 & 89,6 & Ictericia & 139 & 95,8 \\
\hline Dolor abdominal & 68 & 46,7 & Hepatomegalia & 111 & 76,5 \\
\hline $\begin{array}{l}\text { Dolor hipocondrio } \\
\text { derecho }\end{array}$ & 53 & 36,5 & Esplenomegalia & 15 & 10,3 \\
\hline Sensación febril & 72 & 49,3 & Fiebre: & 61 & 42,0 \\
\hline Vómitos & 43 & 29,6 & Ligera & 56 & 38,6 \\
\hline Anorexia & 61 & 43,0 & Mediana & 4 & 2,7 \\
\hline Náuseas & 29 & 16,5 & Acentuada & 1 & 0,6 \\
\hline Astenia & 35 & 24,1 & Obnubilación mental & 2 & 1,2 \\
\hline Prurito & 9 & 6,2 & Coma & 2 & 1,2 \\
\hline
\end{tabular}

Los dos casos que presentaron obnubilación mental y los dos que presentaron coma murieron. El grado de ictericia observado en el examen clínico fue 
muy variable; sólo en 15 casos fue muy intenso. En 8 de los 111 casos en que se observó hepatomegalia, esta sobrepasaba tres traveses de dedo por debajo del reborde costal, en la línea medio clavicular; en los casos restantes fue de grado moderado. La esplenomegalia se consignó en 15 casos, pero sólo en tres de ellos fue muy acentuada; uno de estos pacientes tenía antecedentes de malaria.

\section{EXÁMENES DE LABORATORIO}

\section{Pruebas de floculación}

Las reacciones de Hanger, oro coloidal, rojo coloidal, turbidez del timol y agua bidestilada fueron practicadas en 139 de los 145 pacientes con hepatitis infecciosa. En 127 casos, estas pruebas fueron uniforme y marcadamente positivas; en 3 casos, las floculaciones fueron negativas a pesar de que existian va. lores altos de bilirrubinemia $(3,5 ; 4,3 ;$ y $11,4 \mathrm{mg}$. \%). En los 9 casos restantes las reacciones fueron poco uniformes y moderadamente positivas. Todos los casos presentaron ictericia de laboratorio.

\section{Bilirrubinemia}

La determinación de la bilirrubina sanguínea se realizó en 128 casos, obteniéndose los resultados que se apuntan en el cuadro 5

\section{CUADRO 5}

Valores de bilirrubina obtenidos en el suero de 128 pacientes con bepatitis infecciosa

\begin{tabular}{|c|c|}
\hline mg./100 cc. de suero & $\mathrm{N}^{\circ}$ de casos \\
\hline $\begin{array}{rlr}\text { Menos de } & 1 \\
1 & \text { a } & 4 \\
5 & \text { a } & 9 \\
10 & \text { a } & 19 \\
20 & \text { a } & 29 \\
30 & \text { a } & 40\end{array}$ & $\begin{array}{r}3 \\
50 \\
28 \\
32 \\
11 \\
4\end{array}$ \\
\hline
\end{tabular}

De los 11 pacientes que presentaron valores de bilirrubinemia entre 20 y $30 \mathrm{mg}$. por ciento, 4 fallecieron, y de los cuatro comprendidos entre $30 \mathrm{y}$ $40 \mathrm{mg}$. por ciento, fallecieron 2, evidenciando estos datos el mal pronóstico que tienen los altos valores de bilirrubinemia. 


\section{Colesterolemia}

La determinación del colesterol sanguíneo se llevó a cabo en 130 de los 145 pacientes de hepatitis infecciosa; el mayor número de casos presentó valores comprendidos entre 200 y $299 \mathrm{mg}$. por ciento, como se puede ver en el cuadro 6.

\section{CUADRO 6}

Valores de colesterol obtenidos en la sangre de 130 pacientes con bepatitis infecciosa

\begin{tabular}{c|c}
\hline \hline mg. $\%$ & No de casos \\
\hline Menos de 100 & 2 \\
100 a 199 & 46 \\
200 a 299 & 62 \\
300 a 399 & 12 \\
400 a 499 & 8 \\
\hline
\end{tabular}

\section{Fosfatasas alcalinas}

La determinación de fostasas alcalinas se practicó en 30 de los 145 pacientes de hepatitis infecciosa; el mayor número de casos estuvo comprendido en el grupo que tenía entre 0 y 9 unidades Bodansky, como se observa en el cuadro 7.

\section{CUADRO 7}

Unidades Bodansky de fosfatasa alcalina por $100 \mathrm{cc}$. de plasma en 30 casos de bepatitis infecciosa

\begin{tabular}{|c|c|c|}
\hline U. B. $/ 100$ cc. de plasma & No de casos & \\
\hline 9 a 9 & 21 & \\
\hline 10 a 19 & 8 & $\bullet$ \\
\hline 20 a 29 & 1 & \\
\hline
\end{tabular}




\section{Hemograma}

Entre los casos de hepatitis infecciosa se encontraron con frecuencia anemias de diverso grado; siendo este un hallazgo común en nuestro medio hospitalario, lo hemos considerado como un fenómeno concomitante. Solamente consignaremos aquí los valores de leucocitos de 75 casos de hepatitits infecciosa, como puede observarse en el cuadro 8.

\section{CUADRO 8}

$V$ alores de leucocitos por $\mathrm{mm}^{3}$ en 75 casos de hepatitis infecciosa

\begin{tabular}{c|c}
\hline \hline Leucocitos por $\mathrm{mm}^{3}$ & No de casos \\
\hline Menos de 5.000 & 10 \\
5.000 a 10.000 & 58 \\
10.000 a 15.000 & 5 \\
15.000 a 20.000 & 2 \\
\hline
\end{tabular}

MortalidAD

De los 145 casos de hepatitis infecciosa estudiados, fallecieron 7, dando una mortalidad de 4,8 por ciento.

\section{CIRROSIS HEPATICA}

El tiempo de evolución de los casos de cirrosis hepática, va desde pocos días hasta varios años con un promedio de 6,7 meses. Se encontró consignado el antecedente de ictericia sólo en cuatro pacientes, con lapsos de 30 años, 6 años, 40 años y 1 año antes de presentarse los síntomas de cirrosis.

\section{Cuadro Clinico}

En el cuadro 9 se consignan los síntomas clínicos y los signos físicos más sobresalientes en los 95 pacientes con cirrosis hepática.

En algunos de los casos que presentaron ascitis muy marcada, se consigna en la historia clínica la dificultad de explorar el hígado y el bazo; por lo tanto, la incidencia real de hepatoesplenomegalia puede estar alterada por este factor. En ningún caso se consignó la presencia de nevus aracniformes. 


\section{CUADRO 9}

Frecuencia de los sintomas clínicos y los signos físicos en 95 pacientes con cirrosis hepática

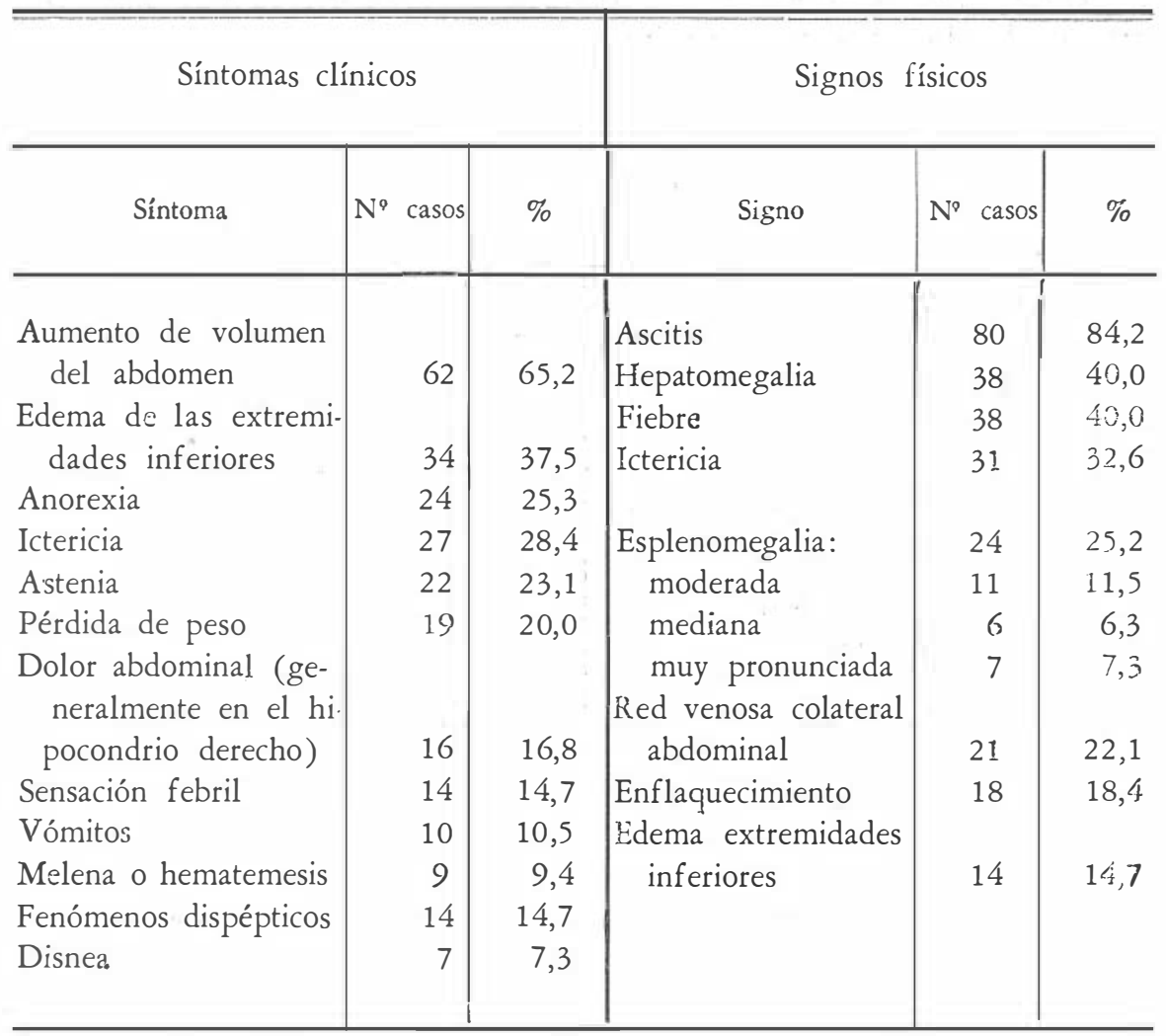

\section{EXÁMENES DE LABORATORIO}

\section{Pruebas de floculación}

Las reacciones de Hanger, oro coloidal, rojo coloidal, turbidez del timol y agua bidestilada, se realizaron en 83 de los 95 casos con cirrosis hepática. De estos 83 pacientes, 76 presentan reacciones uniforme y marcadamente positivas; 6 casos tienen reacciones poco uniformes y moderadamente positivas y en un súlo caso las reacciones fueron uniformemente negativas. En cuatro de los 7 últimos casos, había una ictericia franca de laboratorio.

\section{Bilirrubinemia}

La determinación de la bilirrubina sanguínea se realizó en 78 pacientes (cuadro 10). Debemos señalar que un alto porcentaje dio valores menores de 
$1 \mathrm{mg}$. por ciento. En la mayoría de los casos que presentaron ictericia de laboratorio esta fue de grado moderado.

\section{CUADRO 10}

Valores de bilirrubina obtenidos en el suero de 78 pacientes con cirrosis bepática

\begin{tabular}{|c|c|}
\hline mg./100 cc. de suero & No de casos \\
\hline Menos de 1 & 41 \\
\hline $\begin{array}{lll}1 & \text { a } & 4\end{array}$ & 22 \\
\hline 5 a 9 & 5 \\
\hline 10 a 19 & 5 \\
\hline 20 a 29 & 3 \\
\hline 30 a 39 & 1 \\
\hline 40 o más & 1 \\
\hline & \\
\hline
\end{tabular}

Los valores más altos de $20 \mathrm{mg}$. por ciento se acompañaron de una alta mortalidad; de los últimos 5 casos con bilirrubinemia elevada, fallecieron 4 .

\section{Colesterol}

La determinación cuantitativa del colesterol sanguíneo se practicó en 72 casos, obteniéndose los resultados que se apuntan en el cuadro 11.

\section{CUADRO 11}

Valores de colesterol obtenidos en la sangre de 72 pacientes con cirrosis bepática

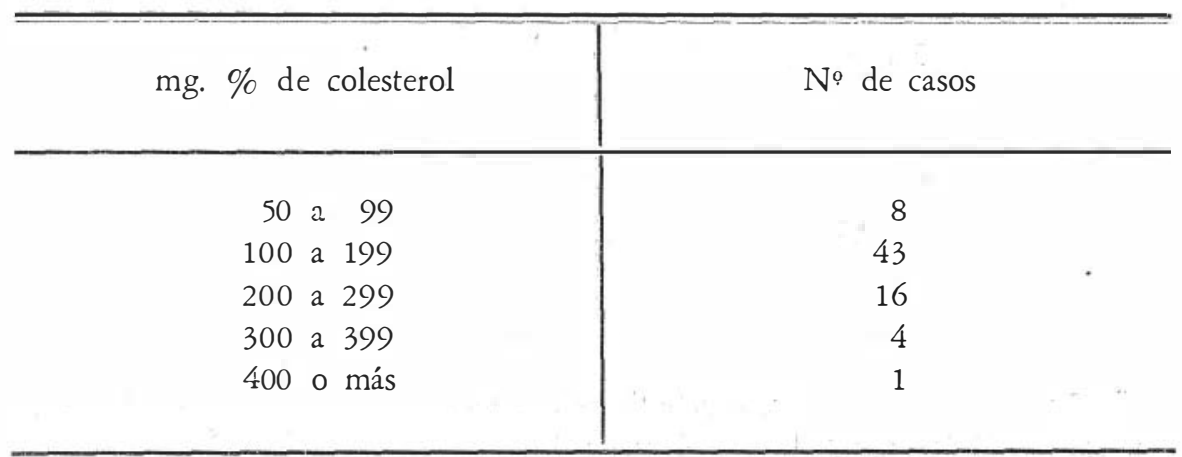




\section{Fosfatasas alcalinas:}

En 31 de los 95 casos de cirrosis hepática se practicó la determinación de fosfatasas alcalinas, obteniéndose la mayor incidencia de casos con menos de 10 unidades Bodansky, como se puede ver en el cuadro 12.

\section{CUADRO 12}

Unidades Bodansky de fosfatasas alcalinas por $100 \mathrm{cc}$. de plasma en 30 casos de cirrosis hepática

\begin{tabular}{c|c}
\hline \hline U.B./100 cc. de plasma & No de casos \\
\hline Menos de 10 & 18 \\
10 a 19 & 11 \\
20 o más & 2 \\
\hline
\end{tabular}

Uno de los casos que presentó más de 20 unidades Bodansky falleció, confirmándose la existencia de una litiasis biliar agregada.

\section{Proteinemia}

La determinación de las proteínas totales se realizó en 47 de los 95 casos de cirrosis hepática y los resultados obtenidos se consignan en el cuadro 13.

CUADRO 13

$V$ alores de proteinas totales en 43 casos de cirrosis hepática

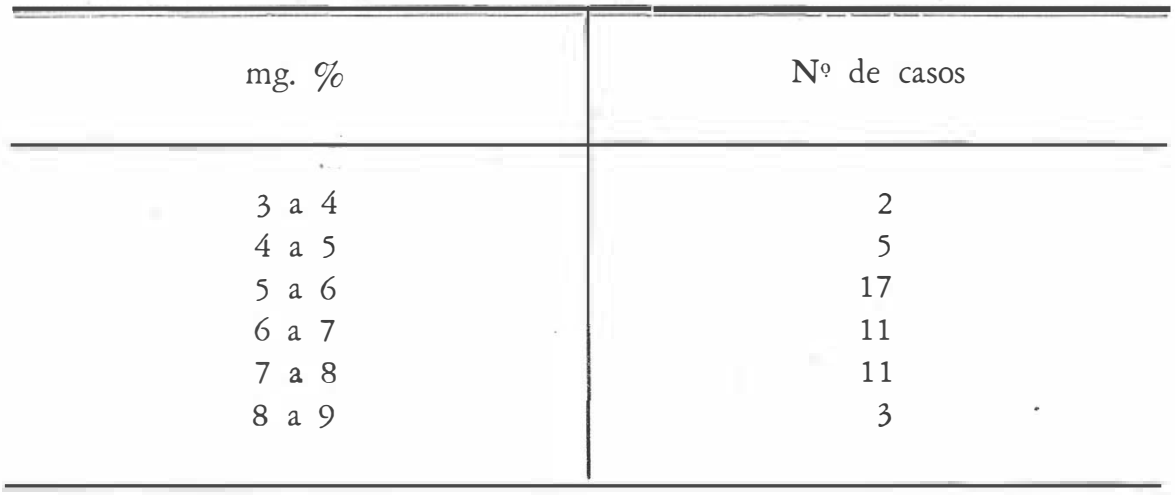

Las seroalbúminas y seroglobulinas se determinaron en 32 casos, obteniéndose los resultados apuntados en el cuadro 14 . 


\section{CUADRO 14}

$V$ alores de seroalbuiminas y seroglobulinas en 32 casos de cirrosis hepática

\begin{tabular}{|c|c|c|c|}
\hline \multicolumn{2}{|c|}{ Seroalbúmina } & \multicolumn{2}{|c|}{ Seroglobulina } \\
\hline $\operatorname{gm~} \%$ & $\mathbf{N}^{e}$ de casos & $\operatorname{gm~} \%$ & No de casos \\
\hline 1 a 2 & 9 & 1 a 2 & 10 \\
\hline 2 a 3 & 16 & 2 a 3 & 3 \\
\hline 3 a 4 & 6 & 3 a 4 & 3 \\
\hline 4 a 5 & 1 & 4 a 5 & 2 \\
\hline - & 一 & 5 a 6 & 3 \\
\hline - & - & 6 a 7 & 9 \\
\hline
\end{tabular}

Las hipoproteinemias, a veces muy marcadas, son bastante frecuentes. Los valores de seroalbúmina son bajos en términos generales; en ocasiones llegan a valores muy bajos (en 9 casos fue menor de $2 \mathrm{gm}$. \% y en 16 menor de $3 \mathrm{gm}$. \%) . Los valores de seroglobulina son normales en términos generales; en algunos casos hubo hiperglobulinemia (i1 casos con más de $4 \mathrm{gm}$. \%).

\section{Hemograma:}

Los valores de glóbulos rojos y hemoglobina se determinaron en 72 pacientes, muchos de los cuales presentaron una anemia severa. En el cuadro 15 se

\section{CUADRO 15}

$V$ alores de eritrocitos y frecuencia de la anquilostomiasis en 72 pacientes con cirrosis bepática

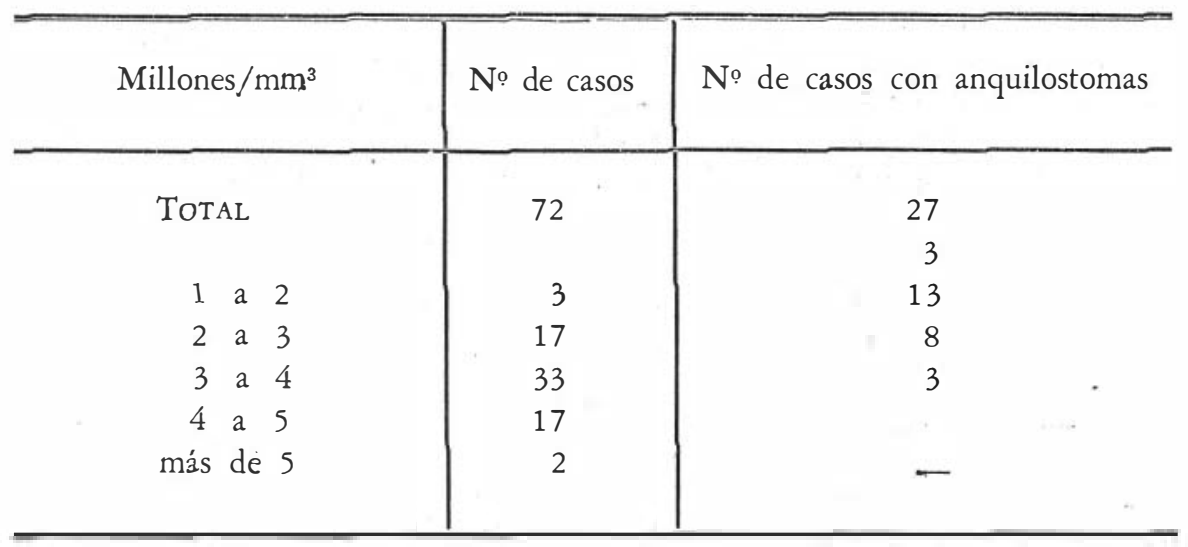


anotan los valores de eritrocitos encontrados en estos pacientes, de los cuales algunos estaban parasitados por anquilostomas.

Es evidente que los pacientes con anemias más severas, presentaron una incidencia más alta de anquilostomiasis, lo cual indica que esta parasitosis juega un papel importante en la anemia de estos cirróticos.

Se practicó recuento leucocitario en 65 pacientes, obteniéndose los resultados que se apuntanı en el cuadro 16.

\section{CUADRO 16}

Valores de leucocitos en 65 pacientes con cirrosis hepática

\begin{tabular}{c|c}
\hline \hline Leucocitos por $\mathrm{mm}^{3}$ & No de casos \\
\hline menos de 5.000 & 20 \\
5.000 a 10.000 & 31 \\
10.000 a 15.000 & 11 \\
15.000 o más & 2 \\
\hline
\end{tabular}

Los dos últimos casos comprendidos en el grupo de más de 15.000 leucocitos por $\mathrm{mm}^{3}$, presentaron valores de 26.000 y 50.000 por $\mathrm{mm}^{3}$.

Llamamos la atención sobre la frecuencia de la leucopenia, 20 casos con menos de 5.000 leucocitos por $\mathrm{mm}^{3}{ }^{3}$, lo cual podría estar en relación con la frecuencia de esplenomegalia en estos pacientes.

\section{MORTALIDAD}

Del total de 95 pacientes que hemos estudiado, fallecieron 37 en el hospital, lo que da una mortalidad de 38,9 por ciento. De estos casos fallecidos en el hospital, se practicó la autopsia en 25 , encontrándose los resultados en el cuadro 17.

\section{CUADRO 17}

Lesiones bepáticas encontradas en 25 casos autopsiados de cirrosis hepática

\begin{tabular}{l|c}
\hline \multicolumn{1}{c|}{ Lesión hépática } & No de casos \\
\hline Cirrosis post-necrótica & 21 \\
Cirrosis Laennec & 2 \\
Cirrosis biliar & 1 \\
Cirrosis post-necrótica con carcinoma & \\
primitivo hepático & 1 \\
\hline
\end{tabular}


Entre el grupo de las cirrosis post-necróticas está incluído un caso que presentó además una tuberculosis caseosa hepatoesplénica.

\section{COMENTARIO}

Un hecho llamativo en el presente trabajo es la mayor frecuencia con que se presentan la hepatitis infecciosa y la cirrosis hepática en ciertos cantones de la Provincia de San José, tales como Mora, Santa Ana, Puriscal y en menor grado Escazú. Estos cantones tienen ciertos factores en común: están en una misma zona geográfica, tienen un nivel sanitario bajo y una alta incidencia de parasitosis intestinal y anemia anquilostomiática. El estado nutritivo de un porcentaje importante de sus habitantes es deficiente. Sin embargo, la relación entre estos factores que acabamos de mencionar y una mayor incidencia de hepatitis infecciosa y cirrosis hepática no está claramente establecida. Desde un punto de vista teórico podría decirse que un mal estado nutricional haría más susceptitbles a los individuos al virus de la hepatitis infecciosa (5), al mismo tiempo que favorecería el desarrollo de una cirrosis a partir de esa hepatitis previa.

Es interesante que las décadas más afectadas en la serie con cirrosis fueron las de 40-49 y 50-59, mientras que en la hepatitis infecciosa fueron grupos de edades inferiores, 20-29 y 30-39. Si esto tiene o no algún significado en el sentido de que la cirrosis daría síntomas clínicos de 10 a 20 años después de contraer la hepatitis responsable, es también un asunto que requiere ulterior investigación.

En otros aspectos el cuadro clínico de la hepatitis infecciosa y cirrosis hepática se ajustó a lo que se sabe sobre estas afecciones. Llamamos la atención sobre el hecho de que en nuestros casos de cirrosis no se señala la presencia de nevus aracniformes, mientras que estos se presentan muy comúnmente en la cirrosis de Laennec (62\% (6) ).

El mal pronóstico de las bilirrubinemias elevadas (sobre $20 \mathrm{mg} . \%$ ) tanto en los casos de hepatitis como de cirrosis se hace evidente al observar la evolución fatal de muchos de estos casos.

Las pruebas de floculación fueron positivas en un porcentaje muy elevado de los casos de cirrosis y hepatitis infecciosa, confirmándose su valor como una ayuda diagnóstica y como una valiosa documentación en aquellos casos en que el diagnóstico clínico no deja lugar a dudas. Por otra parte, una proporción importante de los casos de hepatitis infecciosa y de cirrosis presentaron valores de fosfatasas alcalinas entre $10^{\circ}$ y 20 unidades Bodansky. Por lo tanto, en nuestras condiciones de trabajo, estos valores no son incompatibles con el diagnóstico de cirrosis hepática o hepatitis infecciosa.

La frecuente coexistencia de cirrosis hepática y anemia anquilostomiática de diverso grado (cuadro 15) plantea un problema terapéutico importante. La administración del antihelmíntico correspondiente (tetracloroetileno) implica un riesgo de cuantía no precisada, pues el hígado de estos enfermos está lesionado. Com. batir la anemia sin erradicar los parásitos, o sea con la administración de una dieta adecuada y compuestos ferruginosos, es difícil porque se trata de enfermos 
anoréxicos que frecuentemente toleran mal el sulfato ferroso; y desde luego, la persistencia de los parásitos malogra a veces estos esfuerzos.

El predominio tan pronunciado de las cirrosis post-necróticas en los casos que se autopsiaron es del más alto interés. De acuerdo con las clasificaciones más recientes (5) la cirrosis post-necrótica es la más importante, después de la cirrosis de Laennec.

En nuestro ambiente, la alta incidencia de hepatitis infecciosa y el hecho de que la hepatitis y la cirrosis hepática sean marcadamente más frecuentes en las mismas zonas geográficas, son un apoyo en favor de una relación directa entre hepatitis infecciosa y cirrosis hepática.

En opinión de algunos autores $(5,9)$ la hepatitis infecciosa puede dar lugar a un tipo de cirrosis que es indistinguible, desde el punto de vista anátomo patológico, de la cirrosis portal. Esto estaría de acuerdo con Himswrth (3) quien sostiene que no hay una relación constante entre la etiología de la lesión hepática y el tipo anatómico de la misma. Por lo tanto, queda abierta la posibilidad de que aún los casos que en nuestro medio tiene características anatomo patológico, de la cirrosis portal. Esto estaría de acuerdo con Himsworth (3)

PATEK (5) menciona que parece no haher una relación estrecha entre la intensidad de la hepatitis inicial y la producción o no de cirrosis, y que en muchos casos el ataque original habría sido tan leve como para pasar inadvertido clínicamente. En nuestro grupo de cirróticos el antecedente de ictericia aparece consignado en un número muy bajo de casos. Este aspecto merece desde luego un futuro estudio, que podría llevarse a cabo en nuestro ambiente de cortas distancias y gente sedentaria.

\section{RESUMEN}

Se estudian los protocolos de hospitalización de 145 casos de hepatitis y de 95 casos de cirrosis hepática ingresados al Hospital San Juan de Dios desde 1950 hasta setiembre de 1954.

Se señalan las principales características clínicas y de laboratorio de ambas afecciones, de acuerdo con el análisis del material mencionado.

Los valores muy elevados de bilirrubinemia (sobre $2 \mathrm{mg} . \%$ ) tanto en cirrosis como en hepatitis infecciosa son de muy mal pronóstico y van acompañados de una elevada mortalidad.

De los 145 casos de hepatitis estudiados fallecieron 7, dando una mortalidad de $4,7 \%$.

El estudio de la procedencia de los enfermos revela una mayor incidencia de hepatitis infecciosa y cirrosis hepática, en algunos cantones de la provincia de San José.

De los 95 casos de cirrosis estudiados fallecieron 37 en el hospital, dando una mortalidad de $38,9 \%$. 


\section{SUMMARY}

1. The hospitalization protocols of 145 cases of hepatitis and 95 cases of hepatic cirrhosis registered in Hospital San Juan de Dios, San José, Costa Rica from 1950 through September 1954 are studied.

2. The main clinical and laboratory characteristics of either disease are noted, from analysis of the material in question.

3. High bilirubin values (above $2 \mathrm{mg} . \%$ ) in cirrhosis as well as in infectious hepatitis indicate a bad prognosis and show a high mortality.

4. Seven out of 145 cases of hepatitis died, giving a mortality of $4.7 \%$.

5. The record shows a high incidence of infectious hepatitis and hepatic cirrhosis in certain cantones of San José province.

6. Thirty-seven out of 95 cases of cirrhosis investigated died, giving a mortality of $38.9 \%$.

\section{BIBLIOGRAFIA}

1. BLOOR, W. R.

1916. Determination of cholesterol in blood. J. Biol. Chem., 24:227.

2. DuCCI, H.

1949. Ictericias y laboratorio. 196 pp. Ed. Universidad de Chile, Santiago.

3. Himsworth, H. P.

1950. Lectures on the liver and its diseases. 222 pp. II Ed. Harvard University Press. U. S. A.

4. Malloy, H. T. \& K. A. Evelyn

1937. The determination of bilirrubin with the photoelectric colorimeter. J. Biol. Chem., 119:481.

5. PATEK, A. J. JR.

1956 Cirrbosis of the liver. En Cecil \& Loeb., A textbook of medicine. 786 pp. W. Lippincott Co. Phil. Pa.

6. PATEK A. J. JR.

1956. Cirrbosis of the liver. En Cecil \& Loeb, A textbook of medicine. 1786 pp. W' B. Saunders Co. Phil. Pa.

7. Photovolt Corporation

Lumetron. Clinical photoelectric colorimeter. Model 401-A.

8. Shinowara, G. Y., L. M. Jones \& H. L. Reinhart

1942. The estimation of serum inorganic phosphat and "acid" and "alkaline" phos. phatase activity. J. Biol. Chem. 142:921-933.

9. SPellberg, $M$.

1954. Diseases of the liver. 646 pp. Prune \& Stratton, N. Y. 